Präv Gesundheitsf 2018 $13: 269-271$

https://doi.org/10.1007/s11553-018-0672-z

(c) Springer-Verlag GmbH Deutschland, ein Teil von Springer Nature 2018

CrossMark

\author{
Kevin Dadaczynski ${ }^{1,2} \cdot$ Daniel Tolks ${ }^{2,3}$ \\ 'Fachbereich Pflege und Gesundheit, Hochschule Fulda, Fulda, Deutschland \\ ${ }^{2}$ Zentrum für Angewandte Gesundheitswissenschaften, Leuphana Universität Lüneburg, Lüneburg \\ Deutschland \\ ${ }^{3}$ Institut für Didaktik und Ausbildungsforschung in der Medizin, Klinikum der Ludwig-Maximilians- \\ Universität München, München, Deutschland
}

\title{
Spielerische Ansätze als innovative Kommunikationsstrategie der Gesundheitsförderung und Prävention
}

(Smartphones oder Tablets) dazu geführt, dass Spielanwendungen jederzeit und unabhängig vom Aufenthaltsort verfügbar sind.

Vor diesem Hintergrund ist es nicht verwunderlich, dass die Nutzung digitaler Spiele nicht auf einen kleinen Kreis meist jugendlicher Personengruppen beschränkt ist, sondern sich alters- und geschlechtsübergreifend einer hohen Beliebtheit erfreut. So liegt der Anteil der mindestens gelegentlich Spielenden ab 14 Jahren nach Angaben des Bundesverbands Informationswirtschaft, Telekommunikation und neue Medien e. V. in Deutschland bei $43 \%$ [1], was einer Gesamtzahl von etwa 30 Mio. Bundesbürgern entspricht. Hierbei fällt der Anteil spielender Frauen mit $40 \%$ vergleichbar hoch aus und auch mit Blick auf das Alter wird offensichtlich, dass digitale Spielangebote immer stärker von höheren Altersgruppen (>50 Jahre) genutzt werden $[7,10]$. Derzeit liegt das Alter des „Durchschnittsgamers“ bei 35 Jahren.

Digitales Spielen, so die Erkenntnis, lässt sich also nicht auf ein Randphänomen weniger besonders spielaffiner Personen reduzieren, sondern gehört für einen großen Teil der Menschen zur alltäglichen Freizeitbeschäftigung. Die gesundheitswissenschaftliche Auseinandersetzung mit digitalen Spielen konzentrierte sich lange Zeit v.a. auf die negativen Wirkungen, die mit der Mediennutzung oder die Beschäftigung mit bestimmten Spielgenres auf die Gesundheit angenommen wurden. Exemplarisch zu nennen sind Untersuchungen zum exzessiven Computerspielen oder zur Computerspielabhängigkeit, deren Prävalenz sich für das Kindes- und Jugendalter zwischen $0,9-1,7 \%$ bewegt [8]. Deutlich häufiger untersucht sind die negativen Effekte der Nutzung insbesondere gewaltorientierter Spiele, wobei die Ergebnisse sehr heterogen ausfallen und die oftmals angenommene Schädigungen auf das Sozialverhalten oder auch die Begünstigung affektiver Problematiken bislang nicht bestätigen können [3, 5]. Dieser eher pathogenetisch orientierten Forschungsperspektive steht auf der anderen Seite eine Ausrichtung gegenüber, die sich mit den positiven Aspekten des digitalen Spielens beschäftigt. Hierbei handelt es sich um eine deutliche jüngere Forschungsbewegung, die zwei Schwerpunktsetzungen aufweist:

- Eine zunehmende Anzahl empirischer Studien befasst sich mit den gesundheitsbezogenen Effekten entertainmentorientierter Spiele, also jener Spielanwendungen, die v.a. der Unterhaltung der Nutzenden dienen. Neben positiven Effekten auf das Sozialverhalten, personale Kompetenzen wie Problemlösefähig- mehr wegzudenken. So hat die Entwick
lung leistungsstarker mobiler Endgeräte 
keiten oder die Stressbewältigung [4] weisen erste eher klinisch orientierte Untersuchungsbefunde $u$. a. auf eine Reduktion depressiver Symptomlagen hin [6].

- Abseits der nicht intendierten positiven Wirkungen entertainmentorientierter Spiele beschäftigt sich ein weiterer Forschungsstrang mit der Frage, wie spielerische Anwendungen absichtsvoll für gesundheitsförderliche Zwecke eingesetzt werden können. Im Fokus stehen hier v. a. Interventionskonzepte, die sich spielerischer Ansätze und Mechanismen bedienen und diese für gesundheitsbezogene Zielstellungen nutzbar machen.

Gegenstand des vorliegenden Schwerpunktheftes ist die letztgenannte Perspektive, die seit einigen Jahren unter der Bezeichnung "Games for Health“ Einzug in die internationale Diskussion gehalten hat. In Anlehnung an den aus der Kommunikationswissenschaft stammenden Entertainment-EducationAnsatz werden hierbei Gesundheitsthemen niedrigschwellig in fiktionale Unterhaltungsprogramme integriert. Dabei reicht das Ausmaß der Integrationsleistung von der Anreicherung bestehender Interventionen um klassische Spielmechanismen ("Gamification“, z. B. durch Punkte, Levelstruktur, „Badges“) bis hin zu einer systematischen Verknüpfung eines Spielkonzepts mit explizit formulierten gesundheitsförderlichen Lernzielen und Intentionen („Serious Games for Health"). Während im internationalen Kontext bereits seit einigen Jahren vielfältige Aktivitäten im Bereich Forschung und Praxis unternommen, auf eigens eingerichteten Fachkonferenzen diskutiert und vernetzt sowie in eigenen Publikationsorganen (z.B. Games for Health Journal) veröffentlicht werden (für einen Überblick s. [9]), steckt die Entwicklung im deutschsprachigen Raum noch stärker in den Anfängen. Zwar ist auch hier seit etwa 10 Jahren ein erstarkendes Interesse am spielerischen Ansätzen für gesundheitliche Anliegen festzustellen, jedoch rücken Themen der Gesundheitsförderung und Prävention erst in der jüngeren Vergangenheit ver- stärkt in den Fokus [2]. Bislang mangelt es an einer Infrastruktur, die den hier forschenden und praktisch tätigen Akteuren einen wissenschaftlichen Diskurs und die Vernetzung ermöglichen würden. Das Schwerpunktheft möchte daher dazu beitragen, die Forschung und Praxis der spielerischen Gesundheitsförderung und Prävention sichtbar $\mathrm{zu}$ machen und darüber zu einer interdisziplinären Diskussion anzuregen. Die in diesem Heft aufgenommenen Beiträge spannen dabei einen breiten Bogen, in dem sowohl historische und konzeptionelle Aspekte als auch konkrete Projekte und deren Evaluationsergebnisse zur Sprache kommen.

Den Anfang machen Daniel Tolks et al., die in ihrem Beitrag die zentralen Entwicklungslinien von „Serious Games“ im historischen Kontext bis hin zur Gegenwart zeichnen. Dabei verdeutlichen die Autoren anhand zahlreicher Belege, dass ernsthafte Spiele seit jeher ein integraler Bestandteil der menschlichen Kultur sind und dass bereits in den 1970er-Jahren des letzten Jahrhunderts Spiele für therapeutische Zwecke eingesetzt wurden.

Dieser einführenden Betrachtung schließen sich zwei Beiträge an, welche jeweils eine spezifische Lebensphase in den Blick nehmen. Auf Basis einer Recherche in den App-Stores Google Play und Apple iTunes gibt Claudia Lampert einen Überblick über gesundheitsbezogenen App-Anwendungen für Kinder und Jugendliche, von denen ein Teil $(n=66)$ eine gesundheitsfördernde Intention aufweist und die teilweise in spielerischer Form ungesetzt werden. Im Beitrag von Josef Wiemeyer et al. werden hingegen die Effekte der spielbasierten Förderung von körperlicher Aktivität bei älteren Zielgruppen anhand der Analyse von 10 Reviews und Einzelstudien betrachtet. Dabei lassen sich überwiegend positive Wirkungen für das Gleichgewichtstraining, für die Gang- und Mobilitätsschulung als auch für kognitive Funktionen belegen.

Drei weitere Beiträge illustrieren aktuelle Anwendungsbeispiele für „Serious Games for Health“ oder gamifizierte Interventionen. Sandra Döring et al. stellen eine im Rahmen des Projekts „Stress-
Rekord“ entwickelte Weiterbildung für Führungskräfte vor, die in Form eines "Serious Games“ umgesetzt wird und auf den Erhalt und die Förderung der physischen und psychischen Gesundheit abzielt. Demgegenüber zielt das im Beitrag von Felix Kapp et al. vorgestellte rundenbasierte Multiplayergame „Simkult" auf die Förderung einer Kultur der Prävention in Betrieben. Hierbei werden erste Ergebnisse zur Konzeptevaluation vorgestellt. Beide Anwendungen skizzieren nicht nur unterschiedliche Umsetzungsformen, sondern zeigen ebenfalls, dass sich über spielerische Zugänge verhaltens- wie auch verhältnispräventive Zielstellungen adressieren lassen. Eine stärker verhaltensorientierte Ausrichtung findet sich bei David Horstmann et al., die unter dem Titel „Wellbeing Game“ eine gamifizierte Anwendung zur Steigerung des Wohlbefindens aus Neuseeland und ein Projekt zur Adaptation auf den deutschsprachigen Kontext vorstellen.

Während die Wirkungen von spielerischen Ansätzen zentraler Gegenstand der Evaluation sind, werden Aspekte des Nutzungserlebnisses bislang vergleichsweise selten berichte. Vor diesem Hintergrund berichtet Kevin Dadaczynski die Ergebnisse der Pilotierung der gamifizierten Intervention „Healingo Fit“, bei der sowohl die Nutzungshäufigkeit als auch die "user experience" bei $n=80$ Personen erfasst wurde. Sowohl für die hedonische als auch die pragmatische Qualität ergeben sich positive Urteile, die unabhängig vom Geschlecht und Alter auftreten. Einen Schritt weiter gehen Alexandra Smyth et al., deren Evaluationsdaten von $n=171$ Nutzenden der App „Holidaily“ Belege dafür bietet, dass die „user experience" unter Kontrolle weiterer Variablen einen Einfluss auf den gesundheitlichen Effekt der Intervention hat.

Den Abschluss des Schwerpunktheftes bilden zwei Beiträge, in denen der Blick auf Herausforderungen, Grenzen und die zukünftige Entwicklung geworfen wird. Trotz der in verschiedenen Studien nachgewiesenen positiven Effekte bemängeln Johannes Breuer und Daniel Tolks in ihrem Beitrag die methodische Qualität vieler Studien und benennen in ihrer Darstellung Bereiche, in denen spie- 
lerische Anwendungen weniger geeignet sind. Tom Baranowski bringt abschließend eine internationale Perspektive ein und kommt in seinem Gastkommentar ebenfalls zu dem Ergebnis, dass der aktuelle Stand der Forschung nicht immer dem geltenden "state of the art" qualitativ hochwertiger Forschung entspricht. Es bedarf daher weiterer Bemühungen, die Evidenzbasis spielerischer Anwendungen der Gesundheitsförderung und Prävention zu verbessern, um deren Potenziale besser abzuschätzen und Akzep$\operatorname{tanz} \mathrm{zu}$ fördern.

Auch wenn das vorliegende Schwerpunktheft sicherlich nur einen Ausschnitt der aktuellen Forschung und Praxis spielerischer Ansätze abbildet, hoffen wir hiermit einen Beitrag zur weiteren Diskussionen dieses spannenden Feldes zu leisten. Für die Realisierung des vorliegenden Schwerpunkthefts gilt unser herzlicher Dank der Redaktion in Dresden, den Autorinnen und Autoren, die mit einem Beitrag zu diesem Schwerpunktheft beigetragen haben sowie den Gutachterinnen und Gutachtern, die das Ergebnis kritisch zu bewerten hatten.

\section{Korrespondenzadresse}

\section{Prof. Dr. Kevin Dadaczynski}

Fachbereich Pflege und Gesundheit, Hochschule Fulda

Leipziger Straße 123, 36037 Fulda, Deutschland kevin.dadaczynski@pg.hs-fulda.de

Interessenkonflikt. K. Dadaczynski und D. Tolks geben an, dass kein Interessenkonflikt besteht.

\section{Literatur}

1. Bundesverband Informationswirtschaft, Telekommunikation und neue Medien e. V. (2017) Mobil und vernetzt: Die Gaming-Trends 2017. https:// tinyurl.com/y7wccq2g. Zugegriffen: 1. Sept. 2018

2. Dadaczynski K, Schiemann S, Paulus P (2016) Gesundheit spielend fördern. Potenziale und Herausforderungen von digitalen Spieleanwendungen für die Gesundheitsförderung und Prävention. Beltz Juventa, Weinheim

3. Ferguson CJ (2015) Do angry birds make for angry children? A meta-analysis of video game influences on children's and adolescents' aggression, mental health, prosocial behavior, and academic performance. Perspect Psychol Sci 10:646-666

4. Granic I, Lobel A, Engels CME (2014) The benefits of playing video games. Am Psychol 69:66-78

5. Kühn S, Kugler DT, Schmalen K, Weichenberger M, Witt C, Gallinat J (2018) Does playing violent video games cause aggression? A longitudinal intervention study. Mol Psychiatry.https://doi.org/ 10.1038/s41380-018-0031-7

6. Kühn S, Berna F, Lüdtke T, Gallinat J, Moritz S (2018) Fighting depression: action video game play May reduce rumination and increase subjective and objective cognition in depressed patients. Front Psychol 9:129

7. Paaßen B, Morgenroth T, Stratemeyer M (2017) What is a true gamer? The male gamer stereotype and the marginalization of women in video game culture. Sex Roles 76:421-435

8. Rehbein F, Mößle T, Arnaud N, Rumpf HJ (2013) Computerspiel- und Internetsucht. Der aktuelle Forschungsstand. Nervenarzt 84:569-575

9. Shirong A, Kharrazi H (2018) A state-of-the-Art systematic content analysis of games for health. Games Health J. https://doi.org/10.1089/g4h. 2017.0095

10. Verband der deutschen Games-Branchee. V.(2018) Durchschnittsalter der Gamer in Deutschland steigt auf über 36 Jahre. https://tinyurl.com/ ycb9qbdp.Zugegriffen: 1. Sept. 2018 